\title{
Congenital anomalies and genetic disorders in families of children with central nervous system
}

tumours

Susan M Jones, Peter C Phillips, Patricia T Molloy, Beverly J Lange, Michael N Needle, Jaclyn A Biegel

\begin{abstract}
Medical genetic histories of 165 children with primary central nervous system (CNS) tumours and 4599 relatives of these probands were examined to identify birth defects or genetic disorders that may be associated with the aetiology of CNS tumours. Twelve primary malignancies were found in $329(4 \%)$ of the parents of probands. Two of 99 half sibs but no full sibs had malignancies. Twenty-four percent of families had histories warranting consultation for an inherited disorder or birth defect. Single instances of malformations or genetic disorders were reported in 36 families and several disorders were reported in more than one family, including familial hypercholesterolaemia (4), olivopontocerebellar atrophy (2), and familial abdominal aortic aneurysm (2). Although recurring abnormalities were not identified in probands, it is possible that one or more of the birth defects or genetic disorders observed in probands or relatives may be associated with CNS tumourigenesis.
\end{abstract}

( $\mathcal{F}$ Med Genet 1995;32:627-632)

Recent advances in molecular genetics and cytogenetics have increased our understanding of the genetic basis of cancer. ${ }^{1-3}$ However, although brain tumours are the most common solid tumours in children, the genetic aetiologies of childhood primary central nervous system (CNS) tumours are largely unknown.

Some genetic disorders, particularly the phakomatoses, are associated with an increased incidence of CNS tumours. Furthermore, molecular genetic abnormalities identified in these syndromes are likely to have important implications for our understanding of tumourigenesis. For example, identification and cloning of the neurofibromatosis 1 (NF1) gene $^{4-6}$ has permitted detection of germline mutations in NF1 patients ${ }^{7}$ and somatic mutations of the NF1 gene in sporadic cases of NF1 associated tumours. ${ }^{8}$ Studies of the Von Hippel-Lindau syndrome (VHLS) gene $e^{9-12}$ and RET have shown similar findings. ${ }^{13}$

In contrast to the well characterised genetic predisposition to tumours observed in NF1 and VHLS, the vast majority of childhood CNS tumours are sporadic and develop in children without identified risk factors. ${ }^{14}$ However, as genes for specific disorders are identified, oncology programs will face new issues concerning patient and family risk assessment. Few oncology programs are organised to provide such service, since genetic risk assessment and counselling have traditionally been managed by clinical genetics services. Similarly, few clinical genetics services are prepared to provide genetic counselling to large volumes of patients with different types of malignancies. The identification of genes associated with inherited predisposition to malignancy may result in increased physician referral and patient demand for genetic consultation regarding cancer.

Because of the paucity of knowledge regarding the aetiologies of primary paediatric brain tumours and the relative absence of appropriate models for clinical genetic diagnostic and counselling services in childhood brain tumour patients, the Division of Human Genetics and Molecular Biology and the Division of Oncology at The Children's Hospital of Philadelphia jointly undertook a survey of family histories of children with primary CNS tumours. The primary objective of this study was to identify constellations of single occurrences of congenital anomalies or genetic disorders which could be associated with CNS tumourigenesis. Such associations may help identify areas of the genome in which to search for candidate genes for CNS malignancies. An additional objective was to examine families' use of genetic services when referral for such service was made in the setting of the Neurooncology service. This paper describes the organisation and structure of this project and summarises the findings in 4599 relatives in 165 families.

\section{Materials and methods}

A genetic counsellor met families of children who were followed by the Neuro-oncology Service for primary brain or spinal cord tumours, were not adopted, and did not have NF1. NF1 patients were excluded because the NF1 gene has been identified, the mode of inheritance and recurrence risk of this disorder are known, and families of NF1 patients receive genetic counselling through The Children's Hospital of Philadelphia Neurofibromatosis Clinic. A brief prenatal history of the proband was obtained. Standard medical information was recorded about the patient as well as his/her parents, grandparents, great grandparents, sibs, aunts, uncles, and first cousins. If the history 
Table 1 Identification of increased familial risk of malignancy, birth defects, and genetic disease

(1) Did anyone in the family have a tumour that began in the brain or spinal cord? If so, how old was the person when this was diagnosed?

(2) Did anyone in the family have cancer? If so, at what site(s) did this begin? How old was the person when this was diagnosed?

(3) Did anyone in the family have a benign, or non-cancerous, tumour? If so, where was this tumour? How old was the person when this was diagnosed?

(5) Did anyone in the family have a birth defect or defects? If so, what was the birth defect or defects? What was the cause of this, if known?

(6) Were any of your relatives mentally retarded or "slow"? If so, was the cause of mental retardation investigated? If so, what was the cause, if determined?

(7) Did anyone in the family have seizures or epilepsy?

(8) Did any of the women or partners of any of the men in the family have miscarriages or stillbirths? If so, how many? At what time during the pregnancy did the loss occur? Was the cause of pregnancy loss determined? (For stillborns: Did the fetus have any birth defects?)

(9) Did anyone in the family die in infancy? If so, what was the cause of death?

(10) Did anyone in the family die in childhood or as a teenager? If so, what was the cause of death?

(11) Did anyone in the family die as a relatively young adult, that is, in the $20 \mathrm{~s}$, $30 \mathrm{~s}$, or $40 \mathrm{~s}$ ? If so, what was the cause of death?

(12) Did anyone in the family have a growth disorder, that is, was anyone larger or smaller than expected for family norms? Did anyone have a medical disorder that caused them to be tall or short, other than having or being treated for a brain tumour?

(13) Did anyone in the family have irregularities of the skin such as moles or birthmarks? If so, describe these. Where were they? How many were there?

indicated an area where information about additional relatives was desired, the pedigree was expanded accordingly. However, these additional relatives were not included in data tabulation. In addition to obtaining routine medical information, the genetic counsellor asked a series of questions (table 1) to identify disorders suggestive of an increased familial risk of malignancy, birth defects, or genetic disease. Interviews with families of new patients took place within a month of the diagnosis and with families of established patients during visits for continuing treatment or long term follow up care. Most contacts occurred in the outpatient Neuro-oncology Clinic; some families were seen when their child was admitted to the hospital.

The genetic counsellor reviewed the family history with the attending physician if the pedigree showed an indication for further studies, including a clinical genetics consultation. An intellectually impaired or growth delayed child whose disorder was deemed to be secondary to treatment for malignancy was not considered a candidate for referral. Upon agreement from the physician, either the counsellor or physician explained the indication for genetic consultation to the family. If the parents wished to pursue this, the counsellor facilitated the referral. If the family elected genetic service, the reported diagnosis was confirmed by medical records review. Such verification was not sought if the family declined service. Brief genetic counselling for indications of increased risk for an autosomal recessive disorder in future children because of ethnic background, for example, sickle cell anaemia for black couples, was provided by the genetic counsellor at the time of history taking; such counsellings were not included in data tabulation.

Table 2 Number and percentages of tumours by histology

\begin{tabular}{lcc}
\hline Histology & Number & $\begin{array}{l}\text { Percentage of total } \\
\text { (rounded to nearest } \\
\text { whole number) }\end{array}$ \\
\hline $\begin{array}{l}\text { Primitive neuroectodermal tumour } \\
\text { High grade glioma }\end{array}$ & 61 & 36 \\
$\begin{array}{l}\text { (anaplastic astrocytoma, glioblastoma multiforme) } \\
\text { Low grade glioma }\end{array}$ & 18 & 11 \\
$\begin{array}{l}\text { Brainstlary or pilocytic astrocytoma) } \\
\text { Brainstem glioma (diffuse) }\end{array}$ & 39 & 24 \\
Ependymoma (focal) & 3 & 2 \\
Other & 7 & 4 \\
Total & 21 & 13 \\
\hline
\end{tabular}

\section{Results}

During the 21 months from September 1992 to June 1994, 167 families were asked to provide a family history; 165 families participated. For most families the history was contributed by the mother alone $(62 \%)$; in some families this was provided by both parents $(25 \%)$ or the father alone $(10 \%)$; in a few families this was contributed by the patient or maternal grandmother (3\%). A broad spectrum of primary CNS tumours was represented among these patients (table 2). The distribution of tumour histology in this study is comparable to that in published reports from other centres, ${ }^{15-17}$ with the exception of our higher incidence of primitive neuroectodermal tumours (PNETs). This may reflect referral pattern, as The Children's Hospital of Philadelphia Neuro-oncology Program is a PNET research centre. The 165 families reported a total of 4599 relatives. For probands the average age at diagnosis was 8 years 10 months; the youngest patient was aged 3 months and the oldest patient, with a PNET, was aged 38 years at diagnosis.

Of 329 parents about whom information was available, $12(4 \%)$ were reported to have a malignancy. Six mothers each reported one tumour. The mother of a PNET patient was reported to have died at 27 years of complications of a brain tumour; however, this could not be confirmed, as the family denied permission for records review. Three mothers had breast cancer, one had ovarian cancer, and one had thyroid cancer. Six fathers reported the following tumours: optic pathway glioma; a benign pharyngeal tumour diagnosed at 29 years and subsequently a testicular tumour diagnosed at 30 years; bladder cancer; nonmelanoma skin cancer (2); and an unknown primary malignancy.

No probands had children. No family reported malignancy in any of the 261 full sibs of probands. One family reported that the paternal half sister of a female proband with a subependymoma had died of complications of a neuroblastoma; another family reported that the paternal half brother of a male proband with a pineal germinoma died of complications of testicular cancer. No family showed the classic constellation of tumours associated with the Li-Fraumeni syndrome, characterised by sarcomas, breast cancer, brain tumours, and leukaemia. ${ }^{1819}$

One or more indications for genetic consultation were identified in 39 of the 165 famil- 
Table 3 Indications for genetic consultation in paediatric central nervous system tumour patients and relatives (relatives identified by relationship to patient)

\begin{tabular}{|c|c|c|c|}
\hline \multicolumn{2}{|c|}{ Indication } & \multirow{2}{*}{$\begin{array}{l}\text { Evaluation } \\
\text { Referred for genetic re-evaluation }\end{array}$} & \multirow{2}{*}{$\begin{array}{l}\text { Comments } \\
\text { Neurofibromatosis-Noonan syndrome }\end{array}$} \\
\hline $\begin{array}{l}\text { (A) } \\
(1)\end{array}$ & $\begin{array}{l}\text { Patients with additional medical findings } \\
\text { Neurofibromatosis } 1 \text { and pulmonary stenosis: } \\
\text { r/o neurofibromatosis-Noonan syndrome }\end{array}$ & & \\
\hline (2) & Basal cell naevus syndrome, $r / o$ & Referred for genetic consultation & Declined \\
\hline (3) & Fibrodysplasia ossificans progressiva (FOP) & Previous genetic consultation & Patient has spontaneous mutation for FOP \\
\hline (4) & $\begin{array}{l}\text { Charcot-Marie-Tooth disease (autosomal } \\
\text { dominant, type otherwise unspecified) }\end{array}$ & Previous genetic consultation & Father and brother affected \\
\hline (5) & $\begin{array}{l}\text { a Ehlers-Danlos syndrome type III } \\
\text { b Developmental delay (identified after } \\
\text { initial genetics consultation }\end{array}$ & $\begin{array}{l}\text { Previous genetic consultation } \\
\text { Referred for genetic consultation }\end{array}$ & $\begin{array}{l}\text { Mother affected; maternal grandmother and other } \\
\text { maternal relatives possibly affected }\end{array}$ \\
\hline (6) & Duchenne muscular dystrophy (DMD) & Previous genetic consultation & Mother DMD heterozygote \\
\hline (7) & Fragile X syndrome & Previous genetic consultation & Mother and other maternal relatives fragile $\mathrm{X}$ positive \\
\hline (8) & Multiple minor malformations, undiagnosed & Referred for genetic re-evaluation & $\begin{array}{l}\text { Macrocephaly, autosomal dominant: mother affected } \\
\text { Bitemporal narrowing }\end{array}$ \\
\hline (9) & $\begin{array}{l}\text { Hair patterning anomaly (frontal upsweep) } \\
\text { (same patient as case } 22 \text { ) }\end{array}$ & Referred for genetic consultation & Declined \\
\hline $\begin{array}{l}(10) \\
(11) \\
(12) \\
(13) \\
(14) \\
(15)\end{array}$ & $\begin{array}{l}\text { Hair patterning anomaly (lateral upsweep) } \\
\text { Hair patterning anomaly (vertex scalp curl) } \\
\text { Duplicated cystic left kidney } \\
\text { Third branchial cleft sinus tracts } \\
\text { Preauricular pit } \\
\text { Pyloric stenosis } \\
\text { Pyloric stenosis, fh of }\end{array}$ & $\begin{array}{l}\text { Referred for genetic consultation } \\
\text { Referred for genetic consultation } \\
\text { Referred for genetic consultation } \\
\text { Referred for genetic consultation } \\
\text { Referred for genetic consultation } \\
\text { Referred for genetic consultation }\end{array}$ & $\begin{array}{l}\text { Declined } \\
\text { Declined } \\
\text { Branchio-oto-renal syndrome, possible } \\
\text { Isolated malformation } \\
\text { Declined } \\
\text { Declined }\end{array}$ \\
\hline
\end{tabular}

(paternal uncle affected)

(B) Patients with relatives with birth defects or genetic disorders (fh = family history of)

(16) Wiskott-Aldrich (WA) syndrome, fh of (maternal first cousin once removed affected maternal uncle possibly affected)

(17) Von Hippel-Lindau syndrome (VHLS), th of (father affected)

(18) a Familial hypercholesterolaemia, fh of (mother affected) b Pseudocholinesterase deficiency, th of (mother affected)

(19) Familial hypercholesterolaemia, fh of (maternal grandfather and other relatives affected)

(20) Familial hypercholesterolaemia, fh of (mother and other relatives affected)

(21) Familial hypercholesterolaemia, fh of (maternal grandmother affected)

(22) Olivopontocerebellar atrophy, fh of (paternal grandmother affected)

(same patient as case 9)

(23) Olivopontocerebellar atrophy, th of

(24) Abdominal aortic aneurysm, fh of

(25) Abdominal great aunts, great uncles, and first cousin affected)
(25) aneurysm, fh of (mother and maternal grandmother affected)

(26) Aortic aneurysm, fh of (paternal grandfather affected)

(27) Intracranial aneurysms, fh of (maternal grandmother and great grandmother affected)

(28) Mitral valve prolapse syndrome, fh of, $r / o$ (mother and two sisters affected)

(29) Huntington's disease, th of (paternal grandfather affected)

(30) Adult onset polycystic kidney disease, fh of, r/o (paternal grandmother, great aunt, and great grandmother possibly affected)

(31) Ichthyosis, autosomal dominant, type otherwise unspecified, fh of
(mother and other maternal relatives affected)

(32) a Marfan syndrome, fh of, $r / o$ (maternal aunt and uncle possibly affected) b Sickle cell trait, fh of (mother is sickle haemoglobin heterozygote)

Previous genetic consultation

Neuro-oncologist provided genetic consultation

Referred for genetic consultation

Neuro-oncologist provided genetic consultation

Neuro-oncologist provided genetic

consultation

Neuro-oncologist provided genetic

consultation

Referred for genetic consultation

Referred for genetic consultation

Referred for genetic consultation

Referred for genetic consultation

Referred for genetic consultation

Referred for genetic consultation

Referred for genetic consultation

Referred for genetic consultation

Referred for genetic consultation

Previous genetic consultation

Referred for genetic consultation

Previous genetic consultation

Previous genetic consultation

(father affected)

Sickle cell trait, fh of

(mother affected)

b Down's syndrome, th of

(paternal uncle affected)

(34) Retinitis pigmentosa, fh of

(father and paternal uncle affected)

(35) Trisomy 13, fh of

(stillborn sister affected)

(36) Down's syndrome, maternal fh of $\times 3$

(maternal cousins affected)

(37) Microcephaly and developmental delay, th of (brother affected)

(38) Developmental delay, th of (maternal uncle affected)

(39) Spina bifida, th of

(two sibs affected)

(40) Cleft lip and short fifth fingers, fh of (mother affected)

Previous genetic consultation

Referred for genetic consultation

Referred for genetic consultation

Referred for genetic consultation
Previous genetic consultation

Declined

Datient has spontaneous mutation for FOP

her and brother affected

maternal relatives possibly affected

Mother DMD heterozygote

Mother and other maternal relatives fragile $\mathrm{X}$ positive Macrocephaly, autosomal dominant: mother affected Declined

Declined

Declined

Mother possible WA heterozygote

Patient at $50 \%$ risk to have VHLS allele: has brain tumour not characteristic of VHLS

Declined

Patient's serum cholesterol within normal limits (WNL)

Patient's serum cholesterol WNL

Patient's serum cholesterol WNL

Declined

Declined

Familial abdominal aortic aneurysm, autosomal dominant

Declined

No evidence of Marfan syndrome or other inborn error of connective tissue

Declined

Mitral valve prolapse, autosomal dominant

Declined

Declined

Patient not affected

Declined

Mother's future children at $25 \%$ risk to have haemoglobinopathy if future partner is haemoglobinopathy heterozygote

Parents' future children at $25 \%$ risk to have sickle cell $\beta$ thalassaemia

Parents' future children not at increased risk to have Down's syndrome

Declined

Parents' future children at no greater than $1 \%$ risk to have trisomy 13

Declined

Declined

Declined

Declined

Declined

Referred for genetic consultation Declined ies $(24 \%)$ for a total of 44 indications. Table 3 summarises these. Previous genetic evaluations for 14 of these indications had been performed. In two of these cases ( 1 and 8 ) we determined a need to re-evaluate the original diagnoses.
Case 1 was initially thought to have NF1. Although NF1 patients were not generally included in this study, the presence of pulmonary stenosis in this child prompted referral to rule out the neurofibromatosis-Noonan syndrome. 
Case 8 was a child with multiple minor malformations whose diagnosis remained unknown after initial genetic assessment. Families of two other cases (5 and 32) of the previously counselled group reported a newly identified second indication for genetic evaluation. Case 5 was previously diagnosed with Ehlers-Danlos syndrome type III (ED III), inherited from his intellectually normal mother. Developmental delay in this child was identified after the initial genetic assessment, and at his Neuro-oncology Clinic visit referral was made to the clinical genetics service for re-evaluation. Case 32 was a child whose mother had had genetic counselling for sickle cell trait. She reported relatives with medical findings strongly suggestive of Marfan syndrome. These four families were referred for clinical genetic services, along with the remaining 26 families not having previous genetic consultation. Thus, 30 of the 165 families were referred for genetic services. No referrals were made for advanced maternal age, as no mothers over the age of 35 years who were pregnant or planning pregnancy were seen.

Sixteen of the indications for genetic counselling were for a history in the proband of birth defect(s) or additional medical findings (table 3A). Five patients (cases 3, 4, 5a, 6, 7) and their families had been evaluated by a clinical geneticist or a non-geneticist physician for diagnoses of, respectively, fibrodysplasia ossificans progressiva, autosomal dominant Charcot-Marie-Tooth disease (type otherwise unspecified), ED III, Duchenne muscular dystrophy, and fragile $\mathrm{X}$ syndrome. These families did not require further clinical genetics follow up for these diagnoses. Patients with the remaining 11 indications were referred for initial or repeat genetic consultation; of these, five families $(45 \%)$ elected evaluation. Case 1 was found upon re-evaluation to have the neurofibromatosis-Noonan syndrome. The family of case 2 , a medulloblastoma patient, declined referral to rule out the basal cell naevus syndrome in the patient. The referral was prompted by the mother's report that her daughter had numerous naevi in addition to the medulloblastoma, and that the child's father had numerous naevi and paranoid schizophrenia. No other syndromes associated with tumourigenesis were identified in probands.

Twenty-eight of the indications were for possible increased risk for a birth defect or genetic disorder in living or future family members because of the presence of an affected relative(s) (table 3B). Seven of these families had been evaluated by a clinical geneticist or a nongeneticist physician and did not require further follow up regarding these diagnoses. The remaining families had not had genetic consultation and were referred for such service. The neuro-oncologists provided genetic consultation for the four families (cases 18-21) with histories consistent with familial hypercholesterolaemia (FH), one of the most common autosomal dominant disorders in humans. These probands had normal serum cholesterol levels. Of the remaining 17 families who had not had a genetics consultation, three elected a genetics evaluation.
Most parents who declined a genetics assessment cited as the reason for declining the wish to avoid more medical evaluation and stress. Other reasons included: fear of being identified as having a genetic "responsibility" for the tumour or other disorder; fear of being identified as affected with or at risk for a serious genetic disorder; lack of interest in determination of affected or at risk status for a genetic disorder; inability to obtain cooperation from relatives for the evaluation; preoccupation with other family problems.

One family (case 22) reported that the proband's paternal grandmother had olivopontocerebellar atrophy (OPCA). Another family (case 23) reported that the proband's maternal grandmother's two sisters had this disorder, and that the father of this sibship, who died of lung cancer, had a "neurological disorder". The grandmother herself was reported to have been diabetic at her death aged 60 years of a "heart attack". These diagnoses were not confirmed, as both families declined genetic consultation. In both probands the tumour was a posterior fossa PNET. Four families reported histories consistent with familial hypercholesterolaemia. Two families (cases 24 and 25) reported a history of familial abdominal aortic aneurysm (FAAA). One family (case 24) elected genetic consultation, and the reported diagnosis was confirmed in five relatives in two generations.

\section{Discussion}

The primary objective of this study was to identify paediatric CNS tumour patients with medical or family history findings potentially related to tumour aetiology. Birth defects or genetic disorders present in more than one proband or relative would suggest that cytogenetic and molecular genetic analyses of these people might provide information useful in the localisation of genes associated with CNS tumourigenesis. Such approaches successfully led to the identification of the retinoblastoma gene at $13 \mathrm{q} 14^{20}$ and the Wilms' tumour gene WT- 1 at $11 \mathrm{p} 13^{21}$ and have implicated a Beckwith-Wiedemann syndrome locus on $11 \mathrm{p} 15^{22}$

One strategy for identifying factors related to tumourigenesis has been to look for structural abnormalities in children with cancer. Mehes et $a l^{23}$ reported a significantly increased prevalence of minor anomalies in both children with cancer and their sibs, although no specific association of a given pattern of anomalies with a particular malignancy was identified. Schumacher et $a l^{24}$ found an increased incidence of rib anomalies in 1000 children with some, but not all, types of cancer. Both groups suggested that the malignancy and minor anomalies might be different aspects of a single abnormality in embryogenesis. However, neither study evaluated family histories of probands for genetic disorders or malformation syndromes.

Although we did not necessarily expect to find a common malformation syndrome in probands that was previously unrecognised, we 
hypothesised that by surveying a paediatric CNS tumour population we might identify a rare syndrome or single malformation potentially associated with CNS tumourigenesis. Fifteen of 165 patients in our study population had birth defects(s) or genetic disorders. These appeared to be random and no consistent patterns of associated disorders emerged. Nonetheless, a disorder seen only once in the study population may be aetiologically associated with the tumour. For example, familial adenomatous polyposis (FAP) occurs with an incidence of $1 / 8000$ live births. ${ }^{25}$ With approximately 258000000 people in the United States (US), ${ }^{26}$ there are an estimated 32250 US FAP patients. The gross localisation of the gene, APC (adenomatous polyposis coli), for FAP and the allelic Gardner syndrome (GS) began with cytogenetic identification of an interstitial deletion of chromosome $5 \mathrm{q}$ in a mentally retarded/multiply malformed patient with FAP and a negative family history for FAP. ${ }^{27}$ This permitted linkage studies and deletion mapping of tumours which eventually led to the cloning of APC. ${ }^{2829}$ Nine other patients with FAP or GS, mental retardation, major or minor malformations, and an interstitial deletion of chromosome $5 \mathrm{q}$ have been reported. ${ }^{30-34}$ Ten such patients per 32250 US FAP patients give a ratio of one such syndromic patient for every 3225 US FAP subjects. Thus, even in a large series of FAP patients, it would be most unlikely that even two patients would have a malformation syndrome related to an interstitial deletion of 5q. This example shows that the infrequent occurrence of an associated disorder in a series of cancer patients does not exclude a relationship between the disorder and the malignancy, not does it preclude the use of that disorder in the identification of genes aetiologically associated with the malignancy.

Several disorders (FH, FAAA, OPCA) were reported in more than one family. $\mathrm{FH}$ is an autosomal dominant disorder. In some kindreds FAAA is inherited as an autosomal dominant disorder with age dependent penetrance (McKusick No 100070). ${ }^{35}$ The OPCAs are a heterogeneous group of disorders characterised by progressive ataxia, parkinsonism, and pathological changes in the olivary nuclei, pons, and cerebellum. Many cases are sporadic and may have an acquired rather than genetic aetiology. OPCA has been described in a number of families as an autosomal dominant disorder; inheritance may be autosomal recessive in a minority of families. ${ }^{3637} \mathrm{It}$ is not known whether the probands with family histories of OPCA or familial abdominal aortic aneurysm are heterozygotes for these disorders. Although the multiple occurrences of FH, OPCA, and FAAA in our study population are noteworthy, we cannot be certain that the repetitions represent a constellation or that these disorders confer upon gene carriers an increased risk for CNS tumours. Nonetheless, it is possible that the observed associations are because of a genetic lesion common to both the heritable disorder and the tumour. Of particular interest is that the cerebellum is both a site affected in OPCA and the site of origin of each of these two probands' PNETs. Furthermore, OPCA is a very rare disorder, and its occurrence in two of 165 families ascertained through a proband with a CNS tumour is unusual if one assumes that there is no relationship between OPCA gene(s) and genes aetiologically associated with posterior fossa PNETs.

We have shown that a substantial proportion (24\%) of paediatric CNS tumour patients have medical or family histories that warrant genetic evaluation. This finding is not unexpected, given that the identification of additional indications for genetic consultation when a family history is taken has been previously reported. ${ }^{38}$ In our population most indications related to risk assessment and counselling for disorders with no known association with CNS tumours. Of 11 probands for whom genetic consultations were indicated because of the presence of associated findings, five underwent evaluation. In one case (case 1) establishment of the diagnosis of the neurofibromatosis-Noonan syndrome contributed to the understanding of the aetiology of the tumour, as patients with this disorder have an increased risk of developing CNS tumours. This patient and a possible case of basal cell naevus syndrome were the only probands identified with syndromes associated with CNS tumourigenesis.

Families had been referred for genetic services for only 14 of $44(32 \%)$ indications before contact with the counsellor in the Neurooncology service. The reasons for this underreferral are not known, but may relate to lack of physician access to a thorough family history, the physician's and family's focus on urgent medical issues regarding the tumour, or lack of physician recognition of indications for genetic consultation. The absence of referral was notable for several reasons. First, some of these disorders constituted major health problems, because they either shortened life (for example, familial hypercholesterolaemia), caused significant morbidity (for example, Huntington's disease), or conferred handicap (for example, retinitis pigmentosa). Second, for some disorders accurate recurrence risk assessment (for example, for Down's syndrome) or presymptomatic diagnosis (for example, for abdominal aortic aneurysm) through laboratory analysis or examination was available. Third, for some disorders medical intervention to reduce morbidity in affected subjects could be offered (for example, monitoring of cardiovascular status and use of antihypertensive agents to slow aortic dilatation in Marfan syndrome patients). Families elected genetic assessment for seven of 21 (33\%) indications of possible increased risk for a disorder in living or future family members because of the presence of an affected relative(s). However, four of these families were provided genetic consultation in a convenient manner by their child's physician during Neuro-oncology clinic. If these families had refused consultation arranged through another service, the rate would have been only $14 \%$. In contrast, $45 \%$ of couples whose child warranted genetic consultation because of associated findings elected 
genetic evaluation for their child. This suggests that parents were likely to be uninterested in seeking genetic information with no known relevance to their child's tumour, but were more likely to use such service when it might contribute to understanding of their child's health and, possibly, the cause of the tumour. The degree of malignancy of the tumours in the families declining genetic services did not appear to be different from that of the families electing service, suggesting that this was not a determinant of families' use of genetic services.

This study, designed as a hypothesis generating project, provides a basis for subsequent studies. Future larger series that confirm the observations of the associated disorders noted in our patients would support the possibility that links exist between genes responsible for these disorders and genes associated with tumourigenesis. A larger study also would have more power to identify multiple occurrences of those disorders seen only singly in our population. In addition, findings from the present study suggest that paediatric oncology groups planning to incorporate genetic evaluation as part of a multidisciplinary service will improve use of genetic service by arranging this in the oncology clinic rather than the genetics clinic. Lastly, we have identified a group of probands and relatives whose family histories suggest that cytogenetic and molecular genetic analysis of these people may be useful in the identification of genes associated with CNS tumourigenesis.

This work was supported by the Ethel Brown Foederer Fund for Excellence (JAB, PCP), the National Institutes of Health CA 46274 (JAB), the National Institute of Neurological Dis-
orders and Stroke NS 31102 (PCP), and the Ronald McDonald Children's Charities (PCP, JAB).

1 Eng C, Ponder BR. The role of gene mutations in the genesis of familial cancers. FASEB ₹ 1993;7:910-9.

Knudson AG. Antioncogenes and human cancer. Proc Natl Acad Sci USA 1993;90:10914-21.

3 Kelley MJ, Johnson BE. Genetic mechanisms of solid Kelley MJ, Johnson BE. Genetic mechanisms

4 Cawthon RM, Weiss R, Xu G, et al. A major segment of the neurofibromatosis type 1 gene: cDNA sequence, genomic structure and point mutations. Cell 1990;62: 193-201.

5 Viskochil D, Buchberg AM, Xu G, et al. Deletions and a translocation interrupt a cloned gene at the neurofibromatosis type 1 locus. Cell 1990;62:187-92.

6 Wallace MR, Marchuk DA, Andersen LB, et al. Type 1 neurofibromatosis gene: identification of a large transcrip disrupted in three NF1 patients. Science 1990;249:181-6.

7 Gutmann DH, Collins FS. The neurofibromatosis type 1 gene and its protein product, neurofibromin. Neuron 1993 gene and its

8 Li Y, Bollag G, Clark R, et al. Somatic mutations in the neurofibrom

9 Latif F, Kalman T, Gnarra J, et al. Identification of the von Hippel-Lindau disease tumor suppressor gene. Science 1993;269:1317-20.

10 Yao M, Latif F, Orcutt ML, et al. Von Hippel-Lindau disease: identification of deletion mutations by pulsedfield gel electrophoresis. Hum Genet 1993;92:605-14.

11 Gnarra JR, Tory K, Weng Y, et al. Mutations of the VHL tumour suppressor gene in renal carcinoma. Nature Genet 1994;7:85-90.

12 Shuin $\mathrm{T}$, Kondo $\mathrm{K}$, Torigoe $\mathrm{S}$, et al. Frequent somatic mutations and loss of heterozygosity of the von Hippelmutations and loss of heterozygosity of the von HippelLindau tumor suppressor gene in primary h

13 Goodfellow PJ. Inherited cancers associated with the RET proto-oncogene. Curr Opin Genet Dev 1994;4:446-52.

4 Kuijten RR, Bunin GR. Risk factors for childhood brain tumors. Cancer Epidemiol Biomarkers Prev 1993;2:277-88.

5 Young JL Jr, Ries LG, Silverberg E, Horm JW, Miller RW. Cancer incidence, survival and mortality for children younger than age 15 years. Cancer 1986;58:598-602.

16 Bondy ML, Lustbader ED, Buffler PA, Schull WJ, Hardy RJ, Strong LC. Genetic epidemiology of childhood brain tumors. Genet Epidemiol 1991;8:253-67.

17 Gold EB, Leviton A, Lopez R, et al. The role of family history in risk of childhood brain tumors. Cancers 1994 73:1302-10

$18 \mathrm{Li} \mathrm{FP,} \mathrm{Fraumeni} \mathrm{JF.} \mathrm{Soft-tissue} \mathrm{sarcomas,} \mathrm{breast} \mathrm{cancer} \mathrm{and}$ other neoplasms: a familial syndrome? Ann Intern Med 1969;71:747-52.

$19 \mathrm{Li}$ FP, Fraumeni JF, Mulvihill JJ, et al. A cancer family syndrome in twenty-four kindreds. Cancer Res 1988;48: $5358-62$.

20 Friend S, Bernards R, Rogelj S, et al. A human DNA fragment with properties of the gene that predisposes to retinoblastoma and osteosarcoma. Nature 1986;323: 643-6.

21 Coppes MJ, Campbell CE, Williams BRG. The role of WT1 in Wilms tumorigenesis. FASEB $\mathcal{F} 1993 ; 7: 886-95$

22 Ping AJ, Reeve AE, Law DJ, Young MR, Boenke M, Feinberg AP. Genetic linkage of Beckwith-Wiedemann syndrome to 11p15. Am ₹ Hum Genet 1989;44:720-3.

23 Mehes K, Signer E, Pluss HJ, Muller HJ, Stalder G. Increased prevalence of minor anomalies in childhood malig nancies. Eur f Pediatr 1985;144:243-9.

24 Schumacher R, Mai A, Gutjahr P. Association of rib anomalies and malignancy in childhood. Eur $\mathcal{F}$ Pediatr 1992 151:432-4.

25 Fraumeni JF. Genetic factors. In: Holland JF, Frei E, eds. Cancer medicine. 2nd ed. Philadelphia: Lea \& Feibiger, 1982:5-12.

26 United States Census Bureau. Current population report $P$ 251104: population projections of the United States by age, 251104: population projections of the United States by age, sex, race and Hispanic origin for 2050 . Washing

27 Herrera L, Kakati S, Gibas L, Pietrzak E, Sandberg AA. Gardner syndrome in a man with an interstitial deletion of 5q. Am $₹$ Med Genet 1986;25:473-6.

28 Kinzler KW, Nilbert NC, Su L-K, et al. Identification of FAP locus genes from chromosome 5q21. Science 1991; 253:661-5.

29 Groden J, Thliveris A, Samowitz W, et al. Identification and characterization of the familial adenomatous polyposis coli gene. Cell 1991;66:589-600.

30 Hockey KA, Mulcahy MT, Montgomery P, Levitt S, Deletion of chromosome $5 \mathrm{q}$ and familial adenomatous polyposis. F Med Genet 1989;26:61-8.

31 Kobayashi T, Narahara K, Yokoyama Y, et al. Gardner syndrome in a boy with interstitial deletion of the long arm of chromosome 5. Am $₹$ Med Genet 1991;41:460-3.

32 Cross I, Delhanty J, Chapman P, et al. An interchromosomal insertion causing $5 \mathrm{q} 22$ deletion and familial polyposis coli insertion causing 5q22 deletion and familial polyp

33 Hodoson SV, Coonar AS, Hanson PJV, et al. Two cases of $5 q$ deletions in patients with familial adenomatous of 5q deletions in patients with familial adenomatous polyposis: possible

34 Lindgren V, Bryke CR, Ozcelik T, Yang-Feng TL, Francke $\mathrm{U}$. Phenotypic, cytogenetic and molecular studies of three patients with constitutional deletions of chromosome 5 in the region of the gene for familial adenomatous polyposis Am ₹Hum Genet 1992;50:988-97.

35 McKusick VA. Mendelian inheritance in man. 10th ed. Baltimore: Johns Hopkins University Press, 1992:3.

36 Gilman S, Bloedel JR, Lechtenberg R. Disorders of the cerebellum. Philadelphia, FA Davis Co, 1981:244-50.

37 Rosenberg RN, Grossman A. Hereditary ataxia. Neurol Clin 1989;7:25-36.

38 Rubin SP, Malin J, Maidman J. Genetic counseling before prenatal diagnosis for advanced maternal age: an important medical safeguard. Obstet Gynecol 1983;62:155-9. 